Pesq. Vet. Bras. 29(6):445-451, junho 2009

\title{
Principais processos neoplásicos encontrados em psitacídeos mantidos em cativeiro ${ }^{1}$
}

\author{
Silvia Neri Godoy ${ }^{*}$, Venâncio A. Ferreira Alves ${ }^{3,4}$, Cristina Takami Kanamura ${ }^{5}$ \\ e Eliana Reiko Matushima ${ }^{6}$
}

\begin{abstract}
Godoy S.N., Alves V.A.F., Kanamura C.T. \& Matushima E.R. 2009. [Main neoplasic processes in psittacines birds kept in captivity.] Principais processos neoplásicos encontrados em psitacídeos mantidos em cativeiro. Pesquisa Veterinária Brasileira 29(6):445-451. Instituto Chico Mendes de Conservação da Biodiversidade (ICMBio), Rua Profa. Elizabete Rolim 116, Previdência, São Paulo, SP 05514-080, Brazil. E-mail: silng@uol.com.br

An anatomopathologic study of 130 captivy psittacines from zoos, breeders and rehabilitation centers showed the presence of neoplastic disease in seven birds, totalizing $5.4 \%$ of the cases. The neoplasms were identified like cholangiocarcinoma, cholangioma, lymphoma, haemangiosarcoma and renal cell carcinoma. The cholangiocarcinoma was the tumor most commonly observed. it was present in birds. This study describes the macro and microscopic findings of these tumors; also reports diagnostic methods employed to identify these neoplasms.
\end{abstract}

INDEX TERMS: Neoplasms, birds, Psittacines, pathology.

RESUMO.- Um estudo anatomopatológico envolvendo 130 psitacídeos oriundos de centros de triagens, zoológicos e criadouros, visando a determinação da causa de morte destes animais, revelou a presença de neoplasias em sete animais, totalizando $5,4 \%$ dos casos. Os processos neoplasicos foram caracterizados como colangiocarcinoma, colangioma, linfoma, hemangiossarcoma e carcinoma renal primário, sendo o colangiocarcinoma o mais freqüentemente encontrado, presente em três animais. Este trabalho descreve estas neoplasias, levando em consideração os aspectos macro e microscópicos, meios de diagnósticos e espécies acometidas.

\footnotetext{
${ }^{1}$ Recebido em 26 de junho de 2008.

Aceito para publicação em 23 de janeiro de 2009.

2 Instituto Chico Mendes de Conservação da Biodiversidade (ICMBio), Rua Profa. Elizabete Rolim 116, Previdência, São Paulo, SP 05514080, Brasil. *Autor para correspondência: silng@uol.com.br

3 Instituto Adolfo Lutz (IAL), Av. Dr. Arnaldo 355, Cerqueira César, São Paulo, SP 01246-902, Brasil.

${ }^{4}$ Departamento de Patologia, Faculdade de Medicina, Universidade de São Paulo (USP), Av. Dr. Arnaldo 455, Cerqueira César, São Paulo, SP 01246-903.

${ }^{5}$ Laboratório de Imuno-Histoquímica, Divisão de Patologia, IAL, São Paulo, SP.

${ }^{6}$ Faculdade de Medicina Veterinária e Zootecnia (FMVZ), Universidade de São Paulo (USP), Av. Prof. Dr. Orlando Marques de Paiva 87, Cidade Universitária, São Paulo, SP 05508270. E-mail: ermatush@usp.br
}

TERMOS DE INDEXAÇÃO: Neoplasias, aves, Psitacídeos, patologia.

\section{INTRODUÇÃO}

Diversas são as neoplasias que podem afetar os psitacídeos, acometendo qualquer sistema e caracterizadas por crescimento anormal, incontrolado e progressivo de células em qualquer tecido ou órgão. As neoplasias são consideradas em alguns estudos, 3,8 \% das doenças que mais acometem aves silvestres mantidas em cativeiro (Latimer 1994).

Nos psitacídeos os principais tumores encontrados são lipomas, papilomas, fibrossarcomas, hemangiomas, hemangiossarcomas, colangiocarcinomas, linfomas, leiomiomas, leiomiossarcomas e carcinomas (Latimer 1994).

O diagnóstico das neoplasias depende de sua localização e características macro e microscópicas, entretanto, a determinação da histogênese e diagnóstico definitivo são feitos através da imunoistoquímica com anticorpos específicos para as células pesquisadas.

Este trabalho tem o objetivo de descrever os processos neoplasicos e possíveis meios de diagnósticos, identificados em um estudo anatomopatológico envolvendo 130 psitacídeos mantidos em cativeiro, oriundos de centros de triagens, zoológicos, criadores particulares, e domicílios do Estado de São Paulo. 


\section{MATERIAL E MÉTODOS}

O material utilizado neste trabalho foi oriundo de um estudo anatomopatológico envolvendo 130 psitacídeos mantidos em cativeiro, que vieram a óbito em centros de triagens, zoológicos e criadores. As aves estudadas foram submetidas à necropsia até 24 horas após o óbito, e durante o exame macroscópico, foram colhidos fragmentos de todos os órgãos e conservados em formol a $10 \%$ (Formol $40 \%$, diluição $1: 10$ ) para a fixação dos tecidos. Depois de fixado, o material foi incluído em parafina e foram confeccionadas lâminas histopatológicas, cujos cortes foram corados com hematoxilina-eosina (HE).

Dois animais deste estudo foram submetidos à eutanásia depois de diagnosticado quadro clínico irreversível durante procedimento cirúrgico para a extirpação dos mesmos. Houve o aprofundamento do grau anestésico pelo aumento da concentração de isofluorano pelo aparelho de anestesia inalatória.

Amostras de sangue das câmaras cardíacas foram obtidas logo após a retirada do esterno do animal, através de punção cardíaca com seringa e agulha descartáveis, e mantidas em meio para hemocultura para a realização de exame microbiológico. Estas amostras foram semeadas em meio de agar sangue de carneiro (agar base acrescido de $7 \%$ de sangue de carneiro) e agar Sabouraud-dextrose com cloranfenicol (100mg/l) e incubadas, respectivamente, à $37^{\circ} \mathrm{C}$ em aerobiose por $24-72$ horas e em temperatura ambiente por no mínimo de sete dias. Os microorganismos, quando presentes, foram identificados de acordo com Lennette et al. (1985) e classificados segundo Murray et al. (1999).

Os fragmentos de órgãos foram imersos em álcool etílico $96^{\circ} \mathrm{GL}$ e flambados por três vezes consecutivas. A seguir foram triturados em graal estéril com solução salina e areia estéril, e semeados em caldo infusão cérebro-coração (caldo BHI) incubados à $37^{\circ} \mathrm{C}$ em aerobiose por 24 horas. Após este período foram cultivados em agar sangue de carneiro e ágar Sabourauddextrose com cloranfenicol (100mg/l). Os microorganismos isolados foram identificados e classificados da mesma forma descrita para as amostras de sangue cardíaco.

Após a leitura das lâminas histopatológicas, os quadros suspeitos de neoplasias tiveram o material submetido à reação de imunoistoquímica para a determinação da histogênese destes processos. Secções histológicas do tecido foram obtidas a partir dos blocos de parafina, e submetidas à técnica de imunoperoxidase, método streptavidina-biotina. A recuperação dos epítopos foi feita através de calor, utilizando-se panela de pressão. Os marcadores usados nas reações e suas finalidades estão descritos no Quadro 1.

\section{RESULTADOS}

Entre as 130 aves submetidas à necropsia, sete animais revelaram a presença de processos neoplásicos. Destes,
Quadro 1. Marcadores utilizados nas reações imunohistoquímicas em relação a finalidade. São Paulo, 2007

\begin{tabular}{ll}
\hline \multicolumn{1}{c}{ Marcador } & \multicolumn{1}{c}{ Finalidade } \\
\hline CD68 & Identificar macrófagos \\
CD19, CD20, CD45 & Identificar linfócitos B \\
CD3, CD45RO & Identificar linfócitos T \\
CD31, CD34, Fator VIII & Identificar células endoteliais \\
AE1/AE3, CK19 & Identificar células epiteliais \\
1A4 & Identificar células da musculatura lisa \\
S100 & Identificar histiócitos \\
HHF35 & Identificar células musculares lisas, \\
& esqueléticas e cardíacas \\
Cromogranina & Identificar células endócrinas e neurônios
\end{tabular}

quatro aves apresentaram tumores exclusivamente em fígado, um animal apresentou um quadro de carcinoma renal primário, e duas aves apresentaram neoplasias disseminadas em diversos órgãos. Dentre os tumores exclusivamente hepáticos, três animais apresentaram colangiocarcinoma, e um deles colangioma. Os processos generalizados foram caracterizados como linfoma e hemangiossarcoma.

Destes sete animais, três foram oriundos de domicílios, dois de zoológicos, um de criadouro conservacionista e um de centro de triagem. Os animais provenientes de zoológico, criadouro conservacionista e centro de triagem, recebiam dietas adequadas para a espécie, entretanto não existia histórico anterior sobre o manejo destes animais antes deles chegarem a estes locais.

Apesar de diagnosticadas tais neoplasias, estas nem sempre foram responsáveis diretamente pelo óbito do animal. Os animais que apresentaram linfoma e carcinoma renal primário foram submetidos a eutanásia durante procedimento cirúrgico. A relação entre as espécies, origem, a neoplasia diagnosticada e as causas de morte estão descritas no Quadro 2.

Os resultados das reações imuno-histoquímicas encontram-se descritos no Quadro 3. Todos os exames microbiológicos apresentaram resultados negativos para a presença de fungos e bactérias.

\section{DISCUSSÃO}

Entre os animais que apresentaram quadros hepáticos, em três foram diagnosticados colangiocarcinoma, e em um deles, colangioma. Os tumores hepáticos podem ser originários dos hepatócitos, ductos biliares, tecido conjuntivo ou muscular (Schimidt \& Quesenberry 1997). Co-

\section{Quadro 2. Identificação das espécies estudas em relação as neoplasias encontradas e a causa de} morte. São Paulo, 2007

\begin{tabular}{ccccl}
\hline № & Espécie & Origem & Diagnóstico & Causa de morte \\
\hline 1 & Amazona aestiva & Centro de Triagem & Linfoma & Eutanásia \\
2 & Amazona aestiva & Zoológico & Colangiocarcinoma & Neoplasia, colangiocarcinoma \\
3 & Amazona aestiva & Criadouro Particular & Colangioma & Parasitária, Capillaria sp, Ascaridia sp \\
4 & Amazona aestiva & Domicilio & Colangiocarcinoma & Neoplasia, colangiocarcinoma \\
5 & Amazona aestiva & Domićlio & Hemangiossarcoma & Neoplasia, hemangiossarcoma \\
6 & Amazona aestiva & Zoológico & Colangiocarcinoma & Digestivo, cloaca, obstrução \\
7 & Melopsittacus undulatus & Domicilio & Carcinoma Renal Primário & Eutanásia
\end{tabular}




\section{Quadro 3. Relação dos anticorpos utilizados em relação aos resultados obtidos em cada caso de neoplasia estudado. São Paulo, 2007}

\begin{tabular}{clc}
\hline № & \multicolumn{1}{c}{ Anticorpos testados } & Resultados \\
\hline 1 & CD68, CD45, CD45RO, CK19, CD20, CD34, & Negativos \\
& CD3, S100, AE1/AE3, HHF35 & \\
2 & HHF35 e CD34AE1/AE3 & Negativos/Positivo \\
3 & 1A4 e HHF35AE1/AE3 & Negativos/Positivo \\
4 & 1A4 e HHF35AE1/AE3 & Negativos/Positivo \\
5 & AE1/AE3, HHF35, CD34, CD31, & Negativos/Positivo \\
& cromograninaFVIII & \\
6 & HHF35AE1/AE3 & Negativo/Positivo \\
7 & Não realizado & -
\end{tabular}

langiocarcinoma é um tumor originário do epitélio dos ductos biliares, sendo a neoplasia hepática mais freqüentemente observada em aves mantidas em cativeiro (Latimer 1994).

Os sinais clínicos são pouco freqüentes nos animais acometidos, mas pode haver fraqueza, emagrecimento, hepatomegalia, edemaciação, ataxia, tremores e convulsões (Latimer 1994). Nos animais deste estudo que morreram por colangiocarcinoma, um dos animais apresentou convulsão longa seguida de morte, e outros sintomas digestivos como anorexia e diarréia intermitente.

Macroscopicamente, o animal pode apresentar emagrecimento, e o parênquima hepático estar repleto de nódulos, de tamanho variado, consistência firme, de coloração esbranquiçada a amarronzada. Microscopicamente, visualiza-se alteração marcante na arquitetura do órgão, infiltração difusa por tecido biliar neoplásico, composto por canais de células epiteliais cubóides a colunares, dispostas em arranjos glandulares complexos, com ou sem lúmen, separados um do outro por pequeno septo de tecido conjuntivo. As células neoplásicas são bem diferenciadas, com núcleo hipercromático, associadas a hepatócitos bizarros, multinucleados e poucas mitoses (Godoy 2006). Metástases são raras, mas podem ocorrer no pulmão, rins, cérebro, pleura e serosa do proventrículo (Latimer 1994). Em algumas áreas, o tecido neoplásico mostra-se como uma massa constituída por túbulos, suportada por fina rede de estroma, e geralmente apresenta linfócitos agregados. Ainda podem ser observados atrofia hepatocelular e infiltração gordurosa (Potter et al. 1982, Elangbam \& Panciera 1988, Godoy 2006).

Os animais que morreram decorrentes de tal neoplasia apresentaram durante a necropsia, hepatomegalia associada a discretas nodulações esbranquiçadas distribuídas por todo parênquima em um dos casos, e no outro, grandes nódulos brancos infiltrados no parênquima vinhoso, formando extensas lesões cavernosas (Fig.1). Histologicamente foi possível observar ductos complexos associados à necrose centrolobular, mitoses atípicas, desmoplasia e fibrose (Fig.2). Tal neoplasia foi confirmada com a realização de reação imunoistoquímica, sendo positiva para os anticorpos AE1/AE3, que se mostrou um ótimo marcador para células epiteliais de aves, pois além de marcar as células da lesão, também verificou-se que

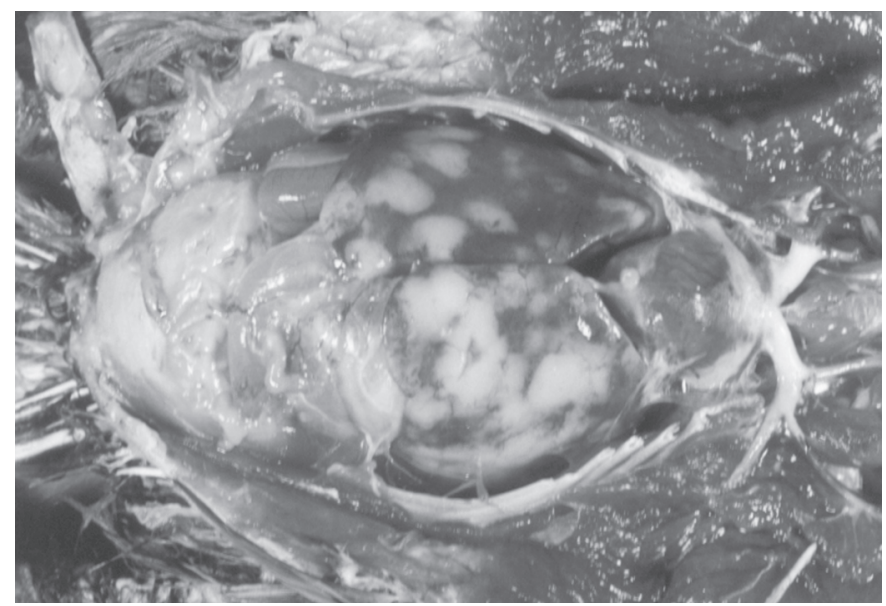

Fig.1. Cavidade abdominal de Amazona aestiva apresentando hepatomegalia associada a múltiplos nódulos brancos infiltrados no parênquima hepático.

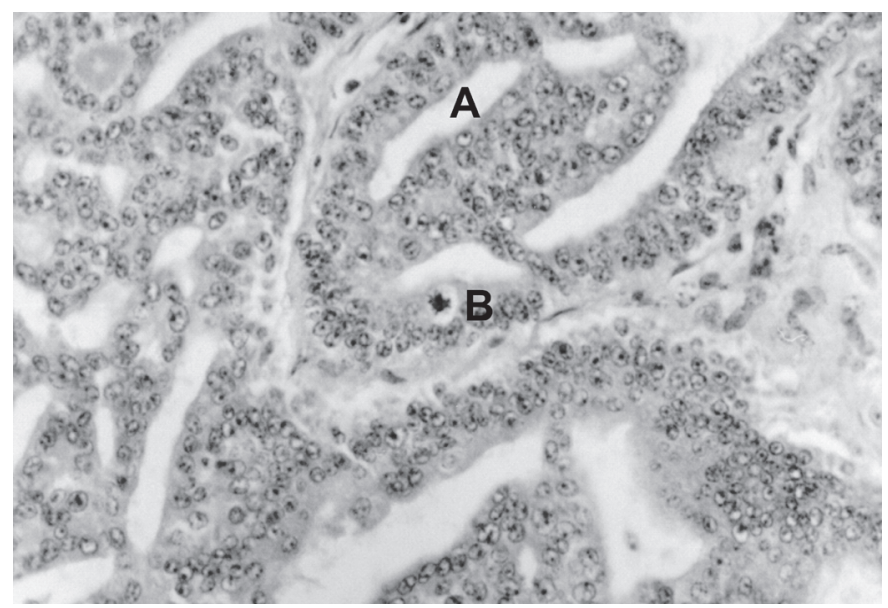

Fig.2. Proliferação de ductos biliares (A), evidenciando a presença de mitoses atípicas (B), no fígado de Amazona aestiva. Quadro compatível com colangiocarcinoma. HE, 1000x.

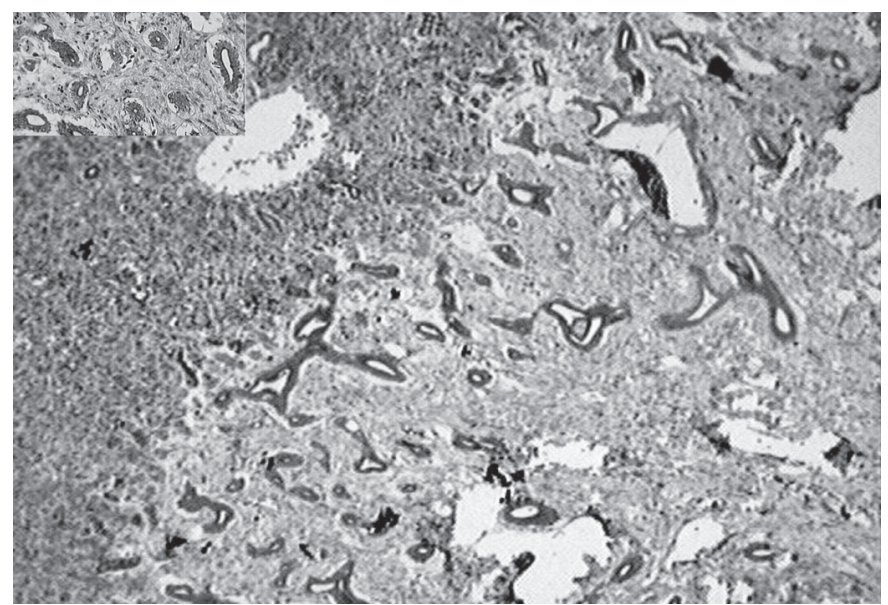

Fig.3. Reação positiva das células epiteliais dos ductos biliares normais e neoplásicos para os anticorpos AE1/AE3, no fígado de Amazona aestiva. Imuno-histoquímica. AE1/AE3, 400x (Detalhe AE1/AE3, 1000x). 


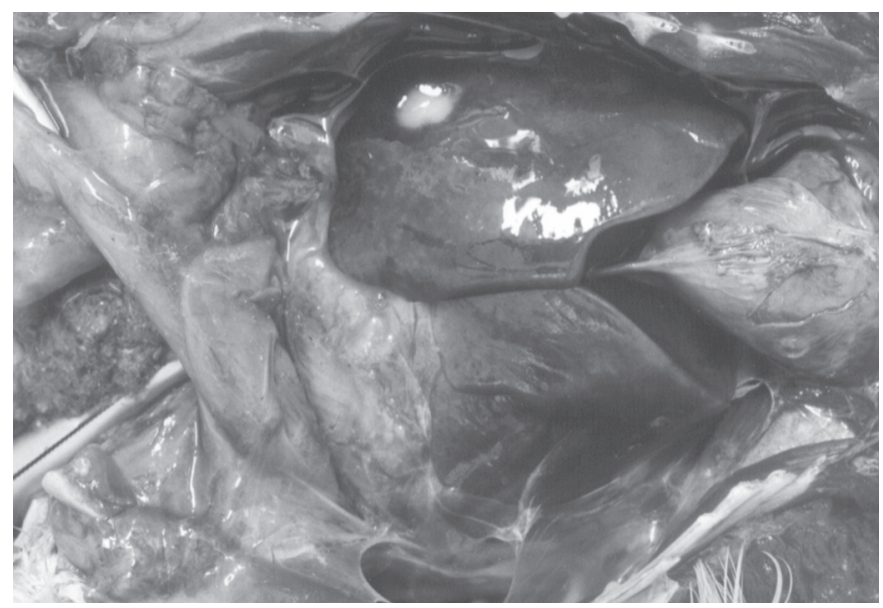

Fig.4. Cavidade abdominal de Amazona aestiva apresentando hepatomegalia associada a nódulo branco infiltrado no parênquima hepático.

os ductos biliares normais estavam fortemente marcados, tanto nestes casos, como nos outros testados (Fig.3).

Os outros dois animais que apresentaram colangioma e colangiocarcinoma como diagnóstico secundário, revelaram durante exame necroscópico apenas um único nódulo esbranquiçado infiltrado no parênquima hepático (Fig.4). À microscopia revelou quadro semelhante aos anteriores, porém focais, e ausência de mitoses aberrantes no colangioma.

Colangiomas são raros quando comparados aos colangiocarcinomas, e podem ocorrer como nódulos únicos ou múltiplos (Latimer 1994). Histologicamente aparecem como estruturas epiteliais tubulares com estroma fibroso denso.

As micotoxinas consumidas através dos alimentos são consideradas carcinogênicas, estando comumente associadas ao aparecimento de neoplasias hepáticas. As micotoxinas são metabólitos químicos produzidos por uma série de fungos que crescem principalmente em grãos e sementes, quando armazenados sob temperatura e umidade inadequadas. A toxina mais comumente associada à doença nas aves é a aflatoxina produzida por AspergiIlus flavus ou $A$. parasiticus, comumente encontrada em grãos e sementes como amendoim, milho e girassol. A aflatoxicose é freqüente em psitacídeos mantidos como aves de companhia que recebem dietas exclusivamente de grãos e sementes. O potencial carcinogênico das micotoxinas depende da ingestão crônica de alimentos contaminados, e inicialmente determinam degeneração e fibrose hepática, com nódulos de regeneração, hiperplasia ductal, esteatohepatite, podendo progredir para uma neoplasia (Godoy 2006).

Entre os animais que apresentaram tumores exclusivamente hepáticos, dois eram oriundos de zoológicos, um de criadouro particular e outro domiciliado. Os animais do zoológico e do criadouro particular recebiam dieta equilibrada para espécie, incluindo frutas, legumes e ração específica. Entretanto, estes animais residiam nestes lo- cais há menos de um ano, e não havia dados sobre a dieta que os mesmos recebiam anteriormente. Porém sabe-se que é extremamente comum o fornecimento de dietas inadequadas, envolvendo grandes porcentagens de sementes, comumente contaminadas por toxinas.

Houve o quadro de prolapso de cloaca em dois animais que apresentaram o diagnóstico de colangiocarcinoma. Apesar de, neste estudo, não terem sido encontradas lesões papilomatosas nesta região, este é um comum achado que muitas vezes estão associadas ou são confundidas com prolapso cloacal. Estes quadros têm sido associados a presença de colangiocarcinoma (Phalen 1998), o que leva a mais um indício da suspeita de papilomatose cloacal.

Papilomas são freqüentemente descritos em aves, répteis e mamíferos e dentre as aves são comuns em psitacídeos, podendo ocorrer em diversos locais do organismo. Muitas das lesões são confinadas à mucosa oral ou cloacal, mas podem ser vistas na conjuntiva, ducto lacrimal nasal, bursa, esôfago, inglúvio, proventrículo e ventrículo (Phalen 1998). As lesões se assemelham ao prolapso retal ou tecido de granulação. Dificilmente é fatal, mas podem diminuir a expectativa de vida dos animais acometidos. As complicações da papilomatose cloacal incluem perda de sangue, desconforto para evacuar, e em casos extremos, obstrução da passagem das fezes e urina (Dvorak et al. 1998, Phalen 1998). Entretanto, alguns autores correlacionam o desenvolvimento de carcinoma de ductos biliares ou pancreáticos nos animais com papilomatose cloacal (Phalen 1998). Existe a suspeita de ser causado por um agente infeccioso, pois quando uma ave doente é introduzida em um aviário, geralmente outras aves contactantes desenvolvem a doença, e lesões semelhantes em humanos têm demonstrado a presença de papilomavírus (Phalen 1998). Papilomavírus já foi demonstrado em papilomas de região perinasal, porém nunca em cloaca (Jacobson et al. 1983, Antinoff \& Hottinger 2000), e partículas de herpesvírus em papilomas cloacais associada à sorologia positiva do animal para tal vírus (Phalen 1998). Os papilomas são caracterizados histologicamente por um epitélio hiperplásico sobre uma base de estroma fibrovascular, associado à figuras de mitose, geralmente nas células basais, e escassas nas células da linha da mucosa (Sundberg et al. 1986, Antinoff \& Hottinger 2000).

Um animal deste estudo revelou a presença de um quadro de hemagiossarcoma, com metástases no fígado, rim e pulmão, confirmado durante a abordagem imunoistoquímica.

O hemangiossarcoma é um tumor que se origina das células endoteliais, formando redes vasculares irregulares, com aspecto cavernoso e repletos de sangue. Pode ser único ou multicêntrico, e pode surgir na pele, fígado, pulmão, baço, músculo, mesentério, rim, coração, oviduto, cápsula testicular, osso ou sinóvia, podendo gerar metástases à distância (Godoy 2006).

Os sinais clínicos dos animais acometidos são variá- 
veis e inespecíficos, incluindo letargia, anorexia, perda de peso, inchaço, dispnéia, hemorragia, petéquias, e eventualmente, quando mediante palpação ou pequenos traumas, podem levar à anemia ou exsangüinação destes tumores (Latimer 1994). O animal deste estudo foi encontrado morto no recinto, sem histórico de sinais clínicos anteriores.

À necropsia, é possível observar nódulos únicos ou múltiplos, de vários tamanhos, vermelho-azulados, edemaciados ou multilobulados, nos diversos tecidos afetados, podendo ou não estar associados a quadros hemorrágicos. Histologicamente aparecem como espaços vasculares que são lineados por células endoteliais distendidas, com núcleo hipercromático associado à presença de mitoses aberrantes (Rossi 1988, Latimer 1994, Suedmeyer 2001). Estas lesões cavernosas, de diversos tamanhos, foram encontradas no fígado, pulmão e rim do animal deste estudo. Ao exame microscópico foi possível visualizar proliferação exuberante de vasos sangüíneos, compostos por células endoteliais pouco diferenciadas e grande número de mitoses aberrantes (Fig.5). Pôde-se observar ainda, múltiplos êmbolos de células tumorais nos vasos sangüíneos do parênquima renal, compostos por células com alto grau de anaplasia e muitas mitoses aberrantes. Este quadro mostrou-se compatível com hemangiossarcoma, mas para um diagnóstico mais preciso, optou-se pela reação de imunoistoquímica, onde os vasos proliferados marcaram-se positivamente para 0 anticorpo anti-fator VIII, já demonstrado pela literatura ser compatível para as células endoteliais de aves (Rossi 1988).

O diagnóstico citológico para hemangiossarcoma, diferente das outras neoplasias, é bastante limitado, pois raramente demonstram células endoteliais, sendo possível apenas observar células sangüíneas. Eritrofagocitose, hemossiderina no interior de macrófagos e cristais de hematoidina podem ser visualizados quando há hemor-

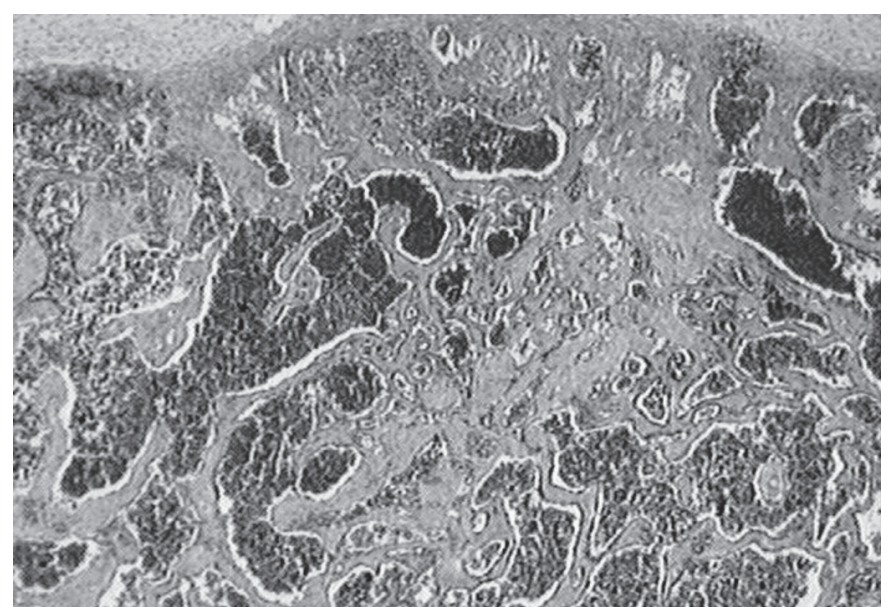

Fig.5. Proliferação exuberante de vasos sangüíneos, compostos por células endoteliais pouco diferenciadas, no fígado de Amazona aestiva. Quadro compatível com hemangiossarcoma. HE, 400x. ragia concomitante. Hematomas, hemangiomas e hemangiossarcomas são difíceis ou impossíveis de serem diferenciados pelo exame citológico (Rossi 1988).

Entre os dois indivíduos que foram submetidos à eutanásia neste estudo, tal procedimento foi realizado visando diminuir o sofrimento de ambos, por apresentarem neoplasias de características extremamente malignas associadas a quadro clínico irreversível. Um dos animais apresentou como histórico a presença de massa em região periocular, que apresentou recidiva após ser extirpada, sendo o animal submetido à eutanásia durante o segundo procedimento cirúrgico para retirada da massa, pois a mesma encontrava-se extremamente infiltrada em região retrobulbar. Após a análise microscópica de tal massa, assim como dos demais órgãos, foi diagnosticado linfoma generalizado, através das características microscópicas do mesmo, apesar dos anticorpos humanos não se mostrarem compatíveis com as células do sistema imune das aves.

O linfoma é um tumor originário de tecido linfóide periférico, e normalmente caracteriza-se pela formação de massas de tecido branco-amarelado ou características sarcomatosas. Normalmente apresenta-se como uma doença multi-sistêmica disseminada, que pode envolver todos os tecidos do corpo, incluindo medula óssea. A exoftalmia pode estar associada a neoplasias retrobulbares como o linfoma, pois existem agregados de células linfóides nesta região (Latimer 1994, Schimidt \& Quesenberry 1997).

As vísceras comumente acometidas pelo linfoma são fígado, baço e rins, sendo raras as manifestações em locais isolados (Latimer 1994, Schimidt \& Quesenberry 1997). No animal deste estudo, os órgãos afetados foram fígado, intestino, baço e massa periocular. Ao exame histopatológico, foi visualizada a presença de células com alto grau de anaplasia e mitoses aberrantes semelhantes a linfócitos alterados, localizados no fígado (Fig 6), baço

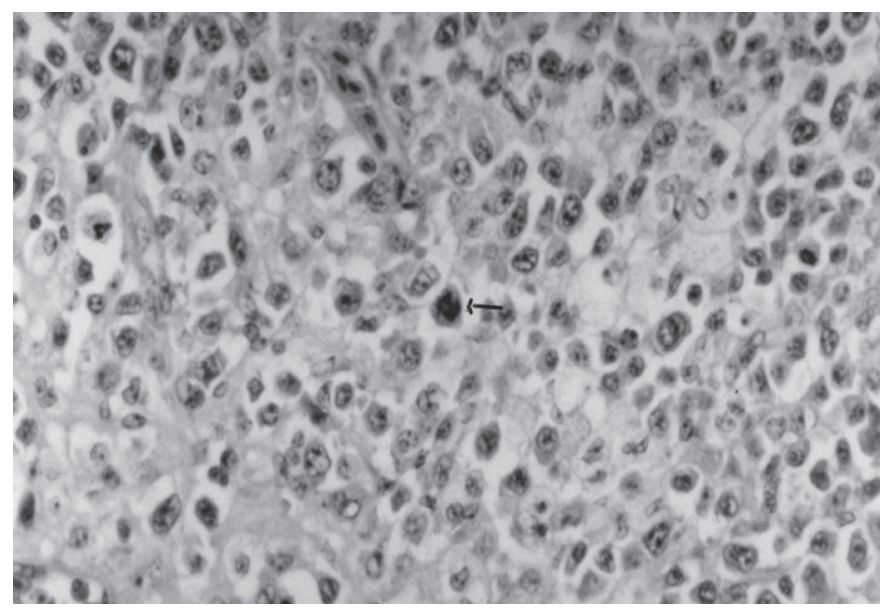

Fig.6. Presença de células com alto grau de anaplasia e mitoses atípicas (seta) semelhantes a linfócitos alterados, no fígado de Amazona aestiva. Quadro compatível com linfoma. HE, 1000x. 
e na massa periorbital, além da proliferação linfóide exuberante nos intestinos, quadro compatível com linfoma. Foram realizadas diversas reações de imuno-histoquímica para a marcação destas células, utilizando-se marcadores desenvolvidos para seres humanos, entretanto nenhuma reação apresentou resultado positivo, o que indica uma provável incompatibilidade de marcadores humanos para as células do sistema imune das aves.

Um diagnóstico presuntivo da neoplasia pode ser efetuado pela presença de inchaço sob a pele ou massas retrobulbares, e a contagem sangüínea demonstrando uma linfocitose e um número aberrante de linfócitos imaturos. Algumas vezes estes quadros podem ser confundidos com hematopoiese extramedular (Latimer 1994).

O outro animal submetido a eutanásia durante o procedimento cirúrgico, foi um periquito australiano, que apresentou durante a laparotomia exploratória, presença de grande massa de aspecto tumoral infiltrada em rins.

Os tumores renais são muito freqüentes em psitacídeos, principalmente em periquitos australianos. O carcinoma renal primário é considerada a neoplasia renal mais freqüentemente vista em animais de cativeiro e de vida livre. É geralmente unilateral, mas pode ocorrer bilateralmente (Hubbart 1983, Lumeij 1985). A etiologia é desconhecida, mas pode ser originário de restos embrionários. Este tipo de neoplasia pode se infiltrar em músculos e ossos adjacentes, podendo se estender até a coluna vertebral e metastatizar em fígado e oviduto (Hubbart 1983, Lumeij 1985).

A maioria dos animais acometidos apresenta paresia, geralmente unilateral (como o animal deste estudo), aumento de volume abdominal, apatia, e sinais nervosos. Isto ocorre porque os nervos do plexo sacral passam pela porção média do rim, onde são comprimidos ou infiltrados por células neoplásicas. Como os rins estão localizados na fossa renal, neoplasias são difíceis de serem isoladas e extirpadas cirurgicamente (Lumeij 1994).

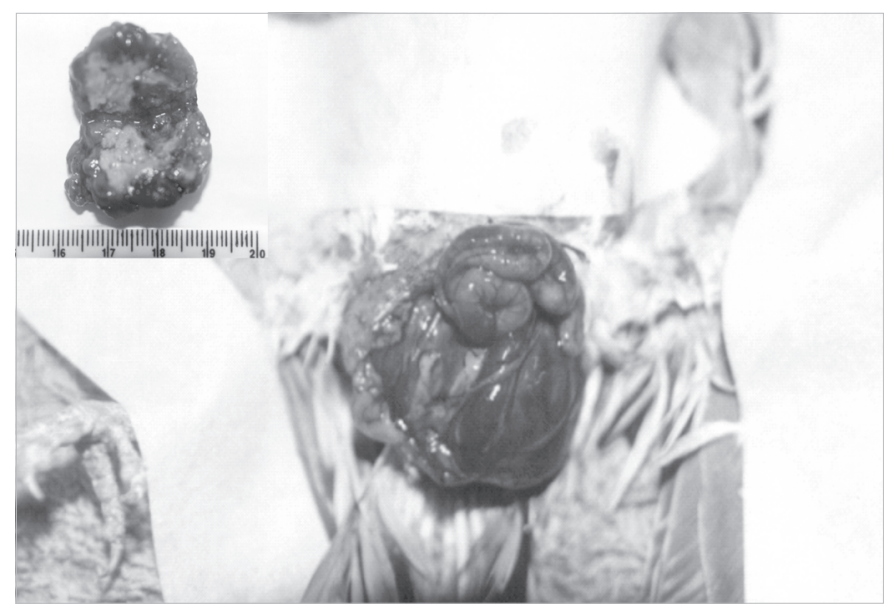

Fig.7. Rins de Melopsittacus undulatus apresentando severo aumento de volume, visualizando ao corte grande massa de coloração amarelada infiltrada em parênquima renal (detalhe).

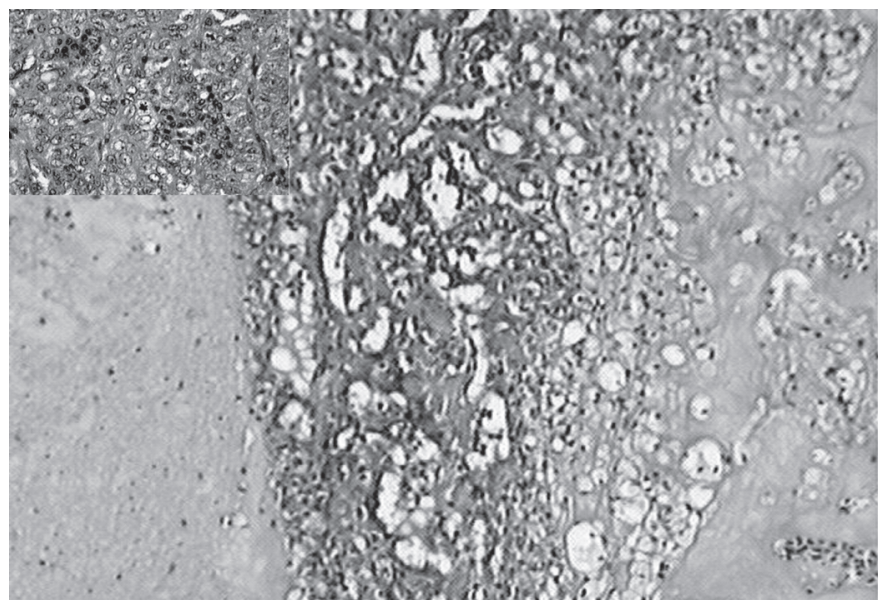

Fig.8. Células gigantes uninucleares, proliferação tubular, extensas áreas de necrose e alto grau de pleomorfismo, no rim de Melopsittacus undulatus. HE, 400x (Detalhe HE, 1000x). Quadro compatível com carcinoma renal.

Os rins afetados apresentam grandes massas multilobuladas, de coloração pálida (Latimer 1994) ou múltiplos focos acinzentados ou nódulos nos rins, de diversos tamanhos, algumas vezes pedunculados, geralmente acometendo apenas um dos rins (Neumann \& Kummerfeld 1983). Neste estudo, o animal apresentou os dois rins acometidos, severamente aumentados, e ao corte visualizou-se grande massa de coloração amarelada infiltrada no parênquima renal (Fig.7). Histologicamente são observadas células epiteliais arranjadas em cordões, túbulos ou ninhos, associadas a mitoses aberrantes, células gigantes multinucleadas, alto grau de pleomorfismo entre as células, proliferação tubular e extensas áreas de necrose. O estroma fibrovascular geralmente é proeminente. O animal deste estudo apresentou microscopicamente, células gigantes uninucleares, proliferação tubular, extensas áreas de necrose e alto grau de pleomorfismo associado a mitoses aberrantes (Fig.8).

Estes relatos de neoplasias em psitacídeos mantidos em cativeiro tiveram o propósito de descrever as principais neoplasias que acometem estes animais, e propor meios de diagnósticos definitivos para tais processos.

Agradecimentos.- À Fundação de Amparo à Pesquisa (FAPESP) pelo suporte financeiro.

\section{REFERÊNCIAS}

Antinoff N. \& Hottinger H.A. 2000. Treatment of a cloacal papilloma by mucosal stripping in an Amazon Parrot. Proc. Annu. Conf. Assoc. Avian Veterinarians, p.97-100.

Dvorak L., Bennett R.A. \& Cranor K. 1998. Cloacotomy for excision of cloacal papillomas in a Catalina Macaw. J. Avian Med. Surg. 12:1115.

Elangbam C.S. \& Panciera R.J. 1988. Cholangiocarcinoma in a blue fronted Amazon Parrot (Amazona aestiva). Avian Dis. 32:594-596.

Godoy S.N. 2006. Psittaciformes, p.222-251. In: Cubas Z.S., Silva J.R. \& Catão-Dias J.L. (Eds), Tratado de Animais Selvagens - Medicina Veterinária. Editora Roca, São Paulo. 
Hubbart G.E. 1983. Renal carcinoma in a captive Edwards Lory (Trichoglossus haematodus capistratus). J. Wildlife Dis. 19:160-161.

Jacobson E.R., Mladinich C.R., Clubb S., Sundberg J.P. \& Lancaster W.D. 1983. Papiloma-like virus infection in an African Gray Parrot. J. Am. Vet. Med. Assoc. 183:1307-1308.

Latimer K.S. 1994. Oncology, p.642-660. In: Harrison G..J., Harrison L.R. \& Ritchie B.W. (Eds), Avian Medicine: Principles and application. Wingers Publishing, Lake Worth.

Lennette E.H., Spauding L.H. \& Truant J.P. 1985. Manual of Clinical Microbiology. ASM Press, Washington, DC. 1149p.

Lumeij J.T. 1994. Nephrology, p.538-555. In: Harrison G.J., Harrison L.R. \& Ritchie B.W. (Eds), Avian Medicine: Principles and application. Wingers Publishing, Lake Worth.

Murray P.R., Baron E.J.O., Pflaller M.A., Tenover F.C. \& Yolken R.H. 1999. Manual of Clinical Microbiology. ASM Press, Washington, DC. 1773p.

Neumann U. \& Kummerfeld N. 1983. Neoplasm in Budgerigars (Melopsittacus undulatus) clinical, pathomorphological and serological findings with special consideration of kidney tumours. Avian Pathol. 12:353362.

Phalen D.N. 1998. Internal Papillomatosis: A Herpesvirus connection? Proc. Annu. Conf. Assoc. Avian Veterinarians, Aug. 26-28, St Paul, p.45-48.

Potter K., Connor T. \& Gallina A.M. 1982. Cholangiocarcinoma in Yellow Faced Amazon Parrot (Amazona xanthops). Avian Dis. 27:556-558,

Rossi G.A. 1988. A poorly-differentiated hepatic haemangiosarcoma in an Amazona farinosa parrot. Avian Pathol. 27:427-430.

Schimidt R.E. \& Quesenberry K. 1997. Neoplasia, p.590-599. In: Altaman R.B. (Ed.), Avian Medicine and Surgery. W.B. Saunders Company, Philadelphia.

Suedmeyer W.M.K., Witter R.L. \& Bermudez A. 2001. Hemangiosarcoma in a Golden Pheasant (Chrysolophus pictus). J. Avian Med. Surg. 15(2):126-130.

Sundberg J.P., Junge R.E., O'banion M.K., Basgall E.J., Harrison G., Herron A.J. \& Shivaprasad H.L. 1986. Cloacal papillomas in psitacines. Am. J. Vet. Res. 47: 928-932. 